\title{
AN ESTIMATE ON THE RICCI CURVATURE OF A SUBMANIFOLD AND SOME APPLICATIONS
}

\author{
PUI-FAI LEUNG \\ (Communicated by Jonathan M. Rosenberg) \\ Dedicated to Professor Tadashi Nagano on his sixtieth birthday
}

\begin{abstract}
We prove a best possible lower bound on the Ricci curvature of an immersed submanifold in a Euclidean space and apply it to study the size of the Gauss image of a complete noncompact hypersurface with constant positive mean curvature in a Euclidean space.
\end{abstract}

\section{INTRODUCTION}

This paper arises as an attempt to apply the method of B. Palmer to the Euclidean case. In [6] Palmer proved an estimate on the size of the image of the Gauss map of a complete spacelike hypersurface $M^{n}$ with nonzero constant mean curvature in a Minkowski space $E_{1}^{n+1}$. His method consisted of two steps. In the first step Palmer proved that the size of the Gauss image implied a lower bound on the first eigenvalue $\lambda_{1}$ of $M$; the main ingredient was Lemma 1 of [6] , which gave an eigenvalue estimate from below. Palmer's proof given in [6] used the property of Green's function. In $\S 5$ we shall give another proof by using an elementary method due to Cheng [1]. In the second step Palmer observed that for the Minkowski case there is an obvious lower bound for the Ricci curvature of $M$ in terms of the mean curvature $H$, namely,

$$
\text { Ric } \geq-H^{2} / 4 \text {. }
$$

(Note that we do not normalize $H$ and so, for example, the unit sphere $S^{n}$ in the Euclidean space $\mathbb{R}^{n+1}$ will have $H^{2}=n^{2}$.) (1.1) enabled Palmer to apply a theorem of Gage [2] to get an upper bound on $\lambda_{1}$ and by combining this with his first step he obtained his theorem.

In order to apply Palmer's method to the Euclidean case we observe that his first step carries over to this case with straightforward modifications. However the obvious result for the Euclidean case that corresponds to (1.1) is an upper bound

$$
\text { Ric } \leq H^{2} / 4 \text {, }
$$

and so there is no obvious generalization of his second step to the Euclidean case. Now we observe that a necessary condition for the Ricci curvature to be

Received by the editors June 1, 1990.

1991 Mathematics Subject Classification. Primary 53C40. 
bounded below is that the scalar curvature $R$ is bounded below. Conversely (1.2) implies

$$
\text { Ric } \geq R-(n-1) H^{2} / 4,
$$

and so this condition is also sufficient. (Again we do not normalize $R$ and so for $S^{n}$ we have $R=n(n-1)$.) Therefore Palmer's second step also carries over under the additional assumption that the scalar curvature be bounded below.

Clearly (1.3) is far from being best possible. (For example look at $S^{n}$. In fact, the right-hand side of (1.3) is nonpositive for all $n \geq 4$ because of (2.20).) This leads us to study the difference between the two sides of (1.3) in order to find a better estimate. Moreover, our main aim in this paper is to present the best possible lower bound for the Ricci curvature of any submanifold. We shall prove

Theorem 1.1. Let $M^{n}$ be an immersed submanifold in the Euclidean space $\mathbb{R}^{n+p}$ where $p$ denotes the codimension. Let Ric, $R$, and $H$ denote the functions that assign to each point of $M$ the minimum Ricci curvature, the scalar curvature, and the mean curvature respectively of $M$ at the point. Then we have

$$
\text { Ric } \geq R-\frac{(n-1) H^{2}}{4}+\frac{1}{4 n^{2}}\left\{\sqrt{n-1}(n-2)|H|-2 \sqrt{(n-1) H^{2}-n R}\right\}^{2} .
$$

Remark. (1.4) is clearly better than (1.3). In fact, (1.4) is best possible because for the unit sphere $S^{n}$ in $\mathbb{R}^{n+1} \subset \mathbb{R}^{n+p}$ we have equality in (1.4).

We shall deduce Theorem 1.1 as a consequence of the following more general theorem, which will be proved in $\S 3$.

Main Theorem. Let $M^{n}$ be an immersed submanifold of the Riemannian manifold $N^{n+p}$. Let Ric, $S$, and $H$ denote the functions that assign to each point of $M$ the minimum Ricci curvature, the square length of the second fundamental form, and the mean curvature respectively of $M$ at the point. If all the sectional curvatures of $N$ are bounded below by $k$ then

Ric $\geq(n-1) k+\frac{1}{n^{2}}\left\{2(n-1) H^{2}-(n-2)|H| \sqrt{(n-1)\left(n S-H^{2}\right)}-n(n-1) S\right\}$.

In $\S 4$ we shall apply our estimate in (1.4) together with the theorem of Gage to deduce an upper bound for $\lambda_{1}(M)$. This result will be applied in $\S 5$ to study the size of the Gauss image of a complete noncompact hypersurface with nonzero constant mean curvature in $\mathbb{R}^{n+1}$.

Remark. For complete noncompact surface with constant mean curvature in $\mathbb{R}^{3}$, Hoffman, Osserman, and Schoen have obtained the best possible result on the size of the Gauss image. Please refer to their excellent paper [3] for details.

\section{Preliminaries}

We consider a submanifold $M^{n}$ in a Riemannian manifold $N^{n+P}$. We look at a small neighborhood in $N$ around a point on $M$.

Let $\left\{e_{1}, \ldots, e_{n+p}\right\}$ be a local frame of orthonormal vector fields on $N$ such that when restricting on $M,\left\{e_{1}, \ldots, e_{n}\right\}$ are tangent to $M$ and $\left\{e_{n+1}, \ldots\right.$, $\left.e_{n+p}\right\}$ are normal to $M$. 
Let $\left\{\omega^{1}, \ldots, \omega^{n+p}\right\}$ be the corresponding dual frame.

We shall use the following convention in this section on ranges of indices and summation will be taken over repeated indices:

$$
\begin{gathered}
1 \leq A, B, C, \cdots \leq n+p, \\
1 \leq i, j, k, \cdots \leq n, \quad n+1 \leq \alpha, \beta, \cdots \leq n+p .
\end{gathered}
$$

The structural equations on $N$ are

$$
\begin{gathered}
d \omega^{A}=-\sum \omega_{B}^{A} \wedge \omega^{B} \\
d \omega_{B}^{A}=-\sum \omega_{C}^{A} \wedge \omega_{B}^{C}+\frac{1}{2} \sum K_{B C D}^{A} \omega^{C} \wedge \omega^{D},
\end{gathered}
$$

where $\omega_{B}^{A}=-\omega_{A}^{B}$ are the connection forms on $N$ and $K_{B C D}^{A}$ are the components of the Riemannian curvature tensor on $N$.

Restricting on $M$, we have $\omega^{\alpha}=0$ and therefore

$$
0=d \omega^{\alpha}=-\sum \omega_{i}^{\alpha} \wedge \omega^{i}
$$

Hence by Cartan's lemma, we have

$$
\omega_{i}^{\alpha}=\sum h_{i j}^{\alpha} \omega^{j}, \quad h_{i j}^{\alpha}=h_{j i}^{\alpha} .
$$

The Riemannian connection of $M$ is defined by $\left(\omega_{j}^{i}\right)$, and the structural equations on $M$ are

$$
d \omega^{i}=-\sum \omega_{j}^{i} \wedge \omega^{j}
$$

and

$$
d \omega_{j}^{i}=-\sum \omega_{k}^{i} \wedge \omega_{j}^{k}+\frac{1}{2} \sum R_{j k l}^{i} \omega^{k} \wedge \omega^{l},
$$

where $R_{j k l}^{i}$ are the components of the Riemannian curvature tensor on $M$.

From (2.2), (2.3), and (2.5) we obtain the Gauss equation

$$
R_{j k l}^{i}=K_{j k l}^{i}+\sum\left(h_{i k}^{\alpha} h_{j l}^{\alpha}-h_{i l}^{\alpha} h_{j k}^{\alpha}\right) .
$$

The second fundamental form $B$ is a tensor of type $(1,2)$ defined by

$$
B:=\sum h_{i j}^{\alpha} \omega^{i} \otimes \omega^{j} \otimes e_{\alpha},
$$

and the square length of the second fundamental form is defined by

$$
S:=\sum\left(h_{i j}^{\alpha}\right)^{2} .
$$

The mean curvature vector of $M$ in $N$ is defined by

$$
v:=\sum h_{i i}^{\alpha} e_{\alpha},
$$

and the mean curvature of $M$ in $N$ is

$$
H:=\|v\| .
$$

From now on we impose the following choice in our basis: if $v \neq 0$, we choose $e_{n+1}$ by $v=\|v\| e_{n+1}$. Therefore by (2.9) and (2.10), we have in all cases

$$
H=\sum_{i} h_{i i}^{n+1} \geq 0
$$


and

$$
\sum_{i} h_{i i}^{\beta}=0 \quad \forall \beta \geq n+2
$$

From (2.8) and (2.11) and by applying the Cauchy-Schwarze inequality, we obtain

$$
n S \geq n \sum_{i}\left(h_{i i}^{n+1}\right)^{2} \geq\left(\sum_{i} h_{i i}^{n+1}\right)^{2}=H^{2}
$$

The Ricci curvature in the direction, say $e_{n}$, at a point $x$ on $M$ is

$$
\text { Ric }:=\sum_{i} R_{n i n}^{i}
$$

and so from (2.6), (2.11), (2.12), and (2.14) we have

$$
\mathrm{Ric}=\sum_{i} K_{n i n}^{i}+h_{n n}^{n+1} H-\sum_{\alpha, i}\left(h_{i n}^{\alpha}\right)^{2} .
$$

The scalar curvature of $M$ is defined by

$$
R:=\sum R_{j i j}^{i}
$$

and so from (2.6), (2.11), (2.12), and (2.16) we have

$$
R=\sum K_{j i j}^{i}+H^{2}-S .
$$

Now consider the special case when $N$ is the Euclidean space $\mathbb{R}^{n+p}$. Then we have

$$
K_{B C D}^{A}=0 .
$$

From (2.17) and (2.18) we have

$$
R=H^{2}-S,
$$

and using (2.13) and (2.19) we have

$$
(n-1) H^{2} \geq n R .
$$

Proof of (1.2). For any unit tangent vector $u$ at $x$ on $M$, we choose a basis such that $e_{n}(x)=u$. Then the Ricci curvature in the direction $u$ at $x$ is given by $(2.15)$, and using (2.18) we have

$$
\text { Ric }=h_{n n}^{n+1} H-\sum_{\alpha, i}\left(h_{i n}^{\alpha}\right)^{2} \leq h_{n n}^{n+1} H-\left(h_{n n}^{n+1}\right)^{2} \leq \frac{H^{2}}{4} \text {. Q.E.D. }
$$

\section{Proof of the MAIN THEOREM}

Our main estimate is the following algebraic lemma. 
Lemma 3.1. Let $\left(h_{i j}\right), i, j=1, \ldots, n$, be a symmetric $n \times n$ matrix, $n \geq 2$. Let $\sum h_{i i}=H \geq 0$ and $\sum\left(h_{i j}\right)^{2}=S$. Then

$$
\begin{aligned}
& h_{n n} H-\sum_{i=1}^{n}\left(h_{i n}\right)^{2} \\
& \geq \frac{1}{n^{2}}\left\{2(n-1) H^{2}-(n-2) H \sqrt{(n-1)\left(n S-H^{2}\right)}-n(n-1) S\right\},
\end{aligned}
$$

and equality holds if and only if either $n=2$ or when $n \geq 3,\left(h_{i j}\right)$ is of the form

$$
\left(\begin{array}{cccc}
\frac{H-x}{n-1} & & & 0 \\
& \ddots & & \\
& & \frac{H-x}{n-1} & \\
0 & & & x
\end{array}\right)
$$

with $x=\frac{1}{n}\left(H-\sqrt{(n-1)\left(n S-H^{2}\right)}\right)$.

Proof. We shall use the same method that we used in proving Lemma 1 on $\mathrm{p}$. 78 of [5]. We shall look for the minimum of $h_{n n} H-\sum_{i}\left(h_{i n}\right)^{2}$ among the class of all $n \times n$ symmetric matrices that have the same Trace $=H$ and the same square norm $=S$. To do this we apply the Lagrange multiplier method to

$$
f=h_{n n} H-\sum_{i=1}^{n}\left(h_{i n}\right)^{2}=\min !
$$

subject to the constraints

$$
\sum h_{i i}=H
$$

and

$$
\sum\left(h_{i i}\right)^{2}+2 \sum_{i<j}\left(h_{i j}\right)^{2}=S
$$

where $\left(h_{i j}\right)$ is a symmetric $n \times n$ matrix. Let

$$
g=h_{n n} H-\sum_{i}\left(h_{i n}\right)^{2}+\lambda\left(\sum h_{i i}-H\right)+\mu\left(\sum\left(h_{i i}\right)^{2}+2 \sum_{i<j}\left(h_{i j}\right)^{2}-S\right),
$$

where $\lambda$ and $\mu$ are the Lagrange multipliers. Setting the partial derivatives of $g$ with respect to the variables $h_{i j}$ equal to zero we obtain the system of equations

$$
\begin{gathered}
\lambda+2 \mu h_{i i}=0 \quad \text { for } i=1, \ldots, n-1, \\
H-2 h_{n n}+\lambda+2 \mu h_{n n}=0, \\
4 \mu h_{i j}=0 \text { for } i<j<n, \\
-2 h_{i n}+4 \mu h_{i n}=0 \text { for } i=1, \ldots, n-1 .
\end{gathered}
$$

Now we shall consider three cases. 
Case $1 \mu=0$. In this case we have $\lambda=0$ by (3.5). Therefore (3.6) and (3.8) imply

$$
h_{1 n}=\cdots=h_{n-1 n}=0
$$

and

$$
h_{n n}=\frac{1}{2} H .
$$

Therefore in this case, we have

$$
f=\frac{1}{4} H^{2},
$$

which as we have seen from (1.2) is the maximum.

Case $2 \mu=\frac{1}{2}$. Then (3.5), (3.6), and (3.7) imply

$$
\begin{aligned}
& h_{i i}=-\lambda \quad \text { for } i=1, \ldots, n-1, \\
& h_{n n}=H+\lambda, \\
& h_{i j}=0 \quad \text { for } 1<j<n .
\end{aligned}
$$

Using (3.10), (3.11), and (3.3) we have

$$
(n-2) \lambda=0 \text {. }
$$

If $n=2$ then a direct calculation using (3.10), (3.11), and (3.4) shows that $f=\frac{1}{2}\left(H^{2}-S\right)$. If $n \geq 3$ then (3.13) implies $\lambda=0$, and so by (3.10) and (3.12) we have

$$
h_{i j}=0 \quad \text { for } i, j=1, \ldots, n-1,
$$

and by (3.4) we find that this only occurs when $H^{2} \leq S$ and $f=\frac{1}{2}\left(H^{2}-S\right) \leq 0$. Hence for Case 2, we have

$$
\begin{gathered}
\text { if } n=2 \text { then } f=\frac{1}{2}\left(H^{2}-S\right) \\
\text { and if } n \geq 3 \text { and } H^{2} \leq S \text {, then } f=\frac{1}{2}\left(H^{2}-S\right) \leq 0 \text {. }
\end{gathered}
$$

Case $3 \mu \neq 0$ and $\mu \neq \frac{1}{2}$. Then (3.5), (3.7), and (3.8) imply

$$
\begin{aligned}
& h_{11}=\cdots=h_{n-1 n-1}, \\
& h_{i j}=0 \quad \text { for } i<j<n, \\
& h_{i n}=0 \quad \text { for } i=1, \ldots, n-1 .
\end{aligned}
$$

For convenience we put $h_{n n}=x$. Then (3.3), (3.16), (3.17), and (3.18) imply that $\left(h_{i j}\right)$ is of the form (3.2) where $x$ is to be determined. Using (3.4) we find that $x$ satisfies the quadratic equation

$$
n x^{2}-2 H x+H^{2}-(n-1) S=0 \text {. }
$$

In this case we have

$$
f=H x-x^{2} \text {. }
$$

Solving (3.19) and substituting into (3.20) we find two values for $f$. Using the given condition that $H \geq 0$, we find that the smaller of these two values is

$$
f=\frac{1}{n^{2}}\left\{2(n-1) H^{2}-(n-2) H \sqrt{(n-1)\left(n S-H^{2}\right)}-n(n-1) S\right\},
$$

and this occurs at $x=\frac{1}{n}\left(H-\sqrt{(n-1)\left(n S-H^{2}\right)}\right)$. 
Finally to complete the proof of the lemma we compare (3.14), (3.15), and (3.21). A direct calculation shows that if $n=2$ then (3.14) $=(3.21)$. If $n \geq 3$ and $H^{2}>S$ then (3.15) does not occur, and so (3.21) is the minimum. If $n \geq 3$ and $H^{2} \leq S$ then a direct calculation shows that $(3.15)>(3.21)$ (here we assume that $S>0$ since if $S=0$ then the lemma is trivial). This completes the proof of lemma. Q.E.D.

Remark. We observe that when $H=0,(3.1)$ becomes

$$
-\sum_{i}\left(h_{i n}\right)^{2} \geq-\frac{n-1}{n} S,
$$

which is the result of Lemma 1 on p. 78 of [5].

Proof of the main theorem. Fix an arbitrary point $x_{0}$ in $M$, and we shall prove that (1.5) holds at $x_{0}$. Consider a small neighborhood in $N$ around $x_{0}$ such that the formulas in $\S 2$ are all valid. Take any unit vector $u$ tangent to $M$ at $x_{0}$. We choose our basis such that $e_{n}\left(x_{0}\right)=u$. If $H\left(x_{0}\right)=0$ then the result is already proved in Theorem A on p. 80 of [5]. Therefore we assume $H\left(x_{0}\right) \neq 0$. Now we choose our basis locally so that $e_{n+1}$ is in the same direction of the mean curvature vector, and so we have $H\left(x_{0}\right)>0$. By (2.15) we have that the Ricci curvature in the direction $u$ at $x_{0}$ is

$$
\text { Ric }=\sum_{i} K_{n i n}^{i}+h_{n n}^{n+1} H-\sum_{i}\left(h_{i n}^{n+1}\right)^{2}-\sum_{\alpha \geq n+2, i}\left(h_{i n}^{\alpha}\right)^{2} .
$$

We let

$$
S_{\alpha}:=\sum_{i, j}\left(h_{i j}^{\alpha}\right)^{2} \quad \text { for } \alpha=n+1, \ldots, n+p .
$$

Applying (3.1) to the matrix $\left(h_{i j}^{n+1}\right)$ and using (2.11) we obtain

$$
\begin{aligned}
h_{n n}^{n+1} H & -\sum_{i}\left(h_{i n}^{n+1}\right)^{2} \\
& \geq \frac{1}{n^{2}}\left\{2(n-1) H^{2}-(n-2) H \sqrt{(n-1)\left(n S_{n+1}-H^{2}\right)}-n(n-1) S_{n+1}\right\} .
\end{aligned}
$$

Using (2.12) we can apply (3.22) to the matrices $\left(h_{i j}^{\alpha}\right)$ for $\alpha=n+2, \ldots, n+p$ to obtain

$$
-\sum_{i}\left(h_{i n}^{\alpha}\right)^{2} \geq-\frac{n-1}{n} S_{\alpha} \quad \text { for } \alpha=n+2, \ldots, n+p,
$$

and so

$$
-\sum_{\alpha \geq n+2, i}\left(h_{i n}^{\alpha}\right)^{2} \geq-\frac{n-1}{n} \sum_{\alpha \geq n+2} S_{\alpha}
$$

Now observe that

$$
S=\sum_{\alpha \geq n+1} S_{\alpha}
$$


(3.27) implies that $S_{n+1} \leq S$ and putting this into (3.25) we have

$$
\begin{aligned}
h_{n n}^{n+1} H & -\sum_{i}\left(h_{i n}^{n+1}\right)^{2} \\
\geq & \frac{1}{n^{2}}\left\{2(n-1) H^{2}-(n-2) H \sqrt{(n-1)\left(n S-H^{2}\right)}-n(n-1) S_{n+1}\right\} .
\end{aligned}
$$

Now adding (3.26) and (3.28) and using (3.27) we obtain

$$
\begin{aligned}
h_{n n}^{n+1} H & -\sum_{\alpha \geq n+1, i}\left(h_{i n}^{\alpha}\right)^{2} \\
& \geq \frac{1}{n^{2}}\left\{2(n-1) H^{2}-(n-2) H \sqrt{(n-1)\left(n S-H^{2}\right)}-n(n-1) S\right\} .
\end{aligned}
$$

By the assumption on the sectional curvature of $N$, we have

$$
\sum_{i=1}^{n} K_{\text {nin }}^{i}=\sum_{i=1}^{n-1} K_{\text {nin }}^{i} \geq(n-1) k \text {. }
$$

Substituting (3.29) and (3.30) into (3.23) we obtain (1.5). Q.E.D.

Theorem 3.2. Let $M^{n}$ be an immersed submanifold in the Euclidean space $\mathbb{R}^{n+p}$. Let Ric, $R$, and $H$ denote the functions that assign to each point of $M$ the minimum Ricci curvature, the scalar curvature, and the mean curvature respectively of $M$ at that point. Then we have

$$
\begin{array}{r}
\text { Ric } \geq \frac{1}{n^{2}}\left\{-(n-1)(n-2) H^{2}-(n-2)|H| \sqrt{(n-1)^{2} H^{2}-n(n-1) R}\right. \\
+n(n-1) R\} .
\end{array}
$$

Proof. Substituting $k=0$ and (2.19) into (1.5) we obtain (3.31). Q.E.D.

Proof of Theorem 1.1. A direct calculation shows that the two right-hand sides of (1.4) and (3.31) are equal. Q.E.D.

\section{Application to eigenvalue estimates}

It is well known that a lower bound on the Ricci curvature implies various bounds on the other geometric quantities. In this section we shall consider one of these situations.

Let $M^{n}$ be a complete noncompact Riemannian manifold. Fix an arbitrary point $x$ in $M$. Let $D_{r}$ denote the open geodesic ball of radius $r$ centered at $x$. Let $\lambda_{1}\left(D_{r}\right)$ denote the first eigenvalue of the boundary value problem

$$
\begin{cases}\Delta u+\lambda u=0 & \text { in } D_{r} \\ u=0 & \text { on } \partial D_{r}\end{cases}
$$

where $\Delta$ is the Laplacian on $M$.

By the domain monotonicity principle we have that $r\left\langle t\right.$ implies $\left.\lambda_{1}\left(D_{r}\right)\right\rangle$ $\lambda_{1}\left(D_{t}\right)$ and hence $\lim _{r \rightarrow \infty} \lambda_{1}\left(D_{r}\right)$ exists. This limit is also independent of the 
choice of the center $x$ as can be seen again from the domain monotonicity principle. Therefore the following definition is well defined

$$
\lambda_{1}(M):=\lim _{r \rightarrow \infty} \lambda_{1}\left(D_{r}\right) .
$$

Theorem 4.1. Let $M^{n}$ be a complete noncompact immersed submanifold of the Euclidean space $\mathbb{R}^{n+p}$. Suppose the mean curvature of $M$ satisfies $0 \leq|H| \leq h$ and the scalar curvature of $M$ satisfies $R \geq-n(n-1) a^{2}$ where $h$ and $a^{2}$ are nonnegative constants. Then

$$
\lambda_{1}(M) \leq \frac{(n-1)^{2}}{4 n^{2}}\left\{(n-2) h^{2}+(n-2) h \sqrt{h^{2}+n^{2} a^{2}}+n^{2}(n-1) a^{2}\right\} .
$$

Proof. This follows by applying our Ricci curvature bound (3.31) together with a theorem of Gage [2, Corollary 5.5, p. 909]. Q.E.D.

Corollary 4.2. Let $M^{n}$ be a complete minimal submanifold in $\mathbb{R}^{n+p}$. Suppose the scalar curvature of $M$ satisfies $R \geq-n(n-1) a^{2}$ for some nonnegative constant $a^{2}$. Then

$$
\lambda_{1}(M) \leq \frac{(n-1)^{3} a^{2}}{4} .
$$

Corollary 4.3. Let $M^{n}$ be a complete noncompact submanifold with nonnegative scalar curvature in $\mathbb{R}^{n+p}$. If the mean curvature of $M$ satisfies $H^{2} \leq h^{2}$ for some constant $h$ then

$$
\lambda_{1}(M) \leq \frac{(n-1)^{2}(n-2) h^{2}}{2 n^{2}} .
$$

\section{COMPLETE HYPERSURFACE WITH CONSTANT MEAN CURVATURE IN $\mathbb{R}^{n+1}$}

We shall study the size of the Gauss image of a complete hypersurface with constant mean curvature in $\mathbb{R}^{n+1}$.

The following lemma is due to Palmer [6, Lemma 1, p. 53]. We give here a different proof using the method of Cheng [1].

Lemma 5.1. Let $D$ be a relatively compact smoothly bounded domain on a Riemannian manifold $M^{n}$. Let $\lambda_{1}(D)$ denote the first eigenvalue of the problem

$$
\begin{cases}\Delta u+\lambda u=0 & \text { in } D, \\ u=0 & \text { on } \partial D .\end{cases}
$$

Suppose $f$ is a smooth function on $\bar{D}$ that satisfies $\Delta f \geq 1$ in $D$. Then

$$
\lambda_{1}(D) \geq \frac{1}{\beta-\alpha},
$$

where $\alpha, \beta$ are any lower and upper bounds respectively of $f$ on $\bar{D}$.

Proof. Put $\lambda_{1}=\lambda_{1}(D)$ and let $u$ denote the solution of (5.1) corresponding to $\lambda_{1}$. It is well known that $u$ does not change sign in $D$, and so we may assume $u>0$ in $D$.

Take any constant $a>\beta$. Then the function $u /(a-f)$ is smooth in $D$, continuous in $\bar{D}$, equal to zero on $\partial D$, and positive in the interior of $D$. Hence this function must attain a maximum at some interior point $x_{0}$ in $D$. 
Hence we have

$$
\left(\nabla \frac{u}{a-f}\right)\left(x_{0}\right)=0
$$

and

$$
\left(\Delta \frac{u}{a-f}\right)\left(x_{0}\right) \leq 0,
$$

where $\nabla$ denotes the gradient.

From (5.3) and (5.4) we obtain at $x_{0}$

$$
\frac{\Delta u}{a-f}+\frac{u \Delta f}{(a-f)^{2}} \leq 0 .
$$

Since $\Delta u=-\lambda_{1} u, u\left(x_{0}\right)>0, a>f\left(x_{0}\right) \geq \alpha$, and $\Delta f \geq 1,(5.5)$ implies

$$
\lambda_{1} \geq 1 /(a-\alpha),
$$

and the result follows by letting $a \rightarrow \beta$. Q.E.D.

Now let us consider $M^{n}$ a complete noncompact immersed hypersurface with constant mean curvature $H=h>0$ in $\mathbb{R}^{n+1}$. Then $e_{n+1}$ gives the Gauss map from $M^{n}$ to the unit sphere $S^{n}$.

Suppose the Gauss image of $M^{n}$ lies inside a closed geodesic disc of radius $\theta$ on $S^{n}$ for some $0<\theta<\pi / 2$. Let $a$ denote the position vector of the center of this geodesic disc. Then we have

$$
\cos \theta \leq\left\langle a, e_{n+1}\right\rangle \leq 1 .
$$

It is well known $\left[4\right.$, p. 131] that for hypersurfaces $M$ in $\mathbb{R}^{n+1}$, the following equation holds on $M$

$$
\Delta\left\langle a, e_{n+1}\right\rangle=S\left\langle a, e_{n+1}\right\rangle+\langle a, \nabla H\rangle .
$$

Using $H \equiv h>0,(2.13),(5.6)$, and (5.7) we obtain

$$
\Delta\left\langle a, e_{n+1}\right\rangle \geq \frac{h^{2} \cos \theta}{n}>0
$$

Hence if we let

$$
f:=\frac{n\left\langle a, e_{n+1}\right\rangle}{h^{2} \cos \theta},
$$

then by (5.6) and (5.8) $f$ satisfies on $M$,

$$
\Delta f \geq 1 \quad \text { and } \quad \frac{n}{h^{2}} \leq f \leq \frac{n}{h^{2} \cos \theta} .
$$

Therefore applying Lemma 5.1 to this $f$, we find that on any geodesic ball $D_{r}$ of $M$, we have

$$
\lambda_{1}\left(D_{r}\right) \geq \frac{h^{2} \cos \theta}{n(1-\cos \theta)} .
$$

Hence we obtain

$$
\lambda_{1}(M) \geq \frac{h^{2} \cos \theta}{n(1-\cos \theta)}
$$


Now suppose that the scalar curvature of $M$ satisfies $R \geq-n(n-1) a^{2}$. Then (4.1) is applicable and we have

$$
\lambda_{1}(M) \leq c,
$$

where $c$ denotes the expression on the right-hand side of (4.1).

Combining (5.10) and (5.11) we obtain

$$
\theta \geq \cos ^{-1}\left(\frac{n c}{h^{2}+n c}\right) \text {. }
$$

Hence we have proved

Theorem 5.2. Let $M^{n}$ be a complete noncompact immersed hypersurface of constant mean curvature $h>0$ in the Euclidean space $\mathbb{R}^{n+1}$. If there is a constant $a^{2}$ such that the scalar curvature of $M$ satisfies $R \geq-n(n-1) a^{2}$, then the Gauss image of $M$ cannot lie totally inside a closed spherical cap of radius $\theta$ for any $\theta<\cos ^{-1}\left(\frac{n c}{h^{2}+n c}\right)$ where $c$ is the value given by the right-hand side of (4.1).

\section{ACKNOWLEDGEMENT}

We are much indebted to the paper [6], which started us on the investigations that led to the results contained herein. It is a great pleasure for us to dedicate this paper to Professor Tadashi Nagano, our teacher and friend, on his sixtieth birthday.

\section{REFERENCES}

1. S. Y. Cheng, Spectrum of the Laplacian and its applications to differential geometry, Ph.D. Dissertation, Univ. of California, Berkeley, 1974.

2. M. Gage, Upper bounds for the first eigenvalue of the Laplace-Beltrami operator, Indiana Univ. Math. J. 29 (1980), 897-912.

3. D. Hoffman, R. Osserman, and R. Schoen, On the Gauss map of complete surfaces of constant mean curvature in $\mathbb{R}^{3}$ and $\mathbb{R}^{4}$, Comment. Math. Helv. 57 (1982), 519-531.

4. D. Laugwitz, Differential and Riemannian geometry, Academic Press, 1965.

5. P. F. Leung, Minimal submanifolds in a sphere, Math. Z. 183 (1983), 75-86.

6. B. Palmer, The Gauss map of a spacelike constant mean curvature hypersurface of Minkowski space, Comment. Math. Helv. 65 (1990), 52-57.

Department of Mathematics, National University of Singapore, Kent Ridge, SingaPORE 0511 